\title{
Biomimetic Nanostructured Surfaces with Designer Mechanics and Geometry for Broad Applications
}

\section{Citation}

Epstein, Alexander K., and Joanna Aizenberg. 2009. Biomimetic nanostructured surfaces with designer mechanics and geometry for broad applications. Materials Research Society Symposium Proceedings 1236: 1236-SS09-07.

\section{Published Version}

doi:10.1557/PROC-1236-SS09-07

\section{Permanent link}

http://nrs.harvard.edu/urn-3:HUL.InstRepos:9121284

\section{Terms of Use}

This article was downloaded from Harvard University's DASH repository, and is made available under the terms and conditions applicable to Open Access Policy Articles, as set forth at http:// nrs.harvard.edu/urn-3:HUL.InstRepos:dash.current.terms-of-use\#OAP

\section{Share Your Story}

The Harvard community has made this article openly available.

Please share how this access benefits you. Submit a story.

\section{Accessibility}




\title{
Biomimetic Nanostructured Surfaces with Designer Mechanics and Geometry for Broad Applications
}

Alexander K Epstein and Joanna Aizenberg

Harvard University School of Engineering and Applied Sciences

29 Oxford Street, Cambridge, MA, U.S.A.

\begin{abstract}
A powerful fabrication platform for a wide range of biomimetic, high-aspect-ratio nanostructured surfaces is introduced. The principles of soft lithography are extended into a double-mold replication process, whereby a master topography is mapped onto an elastomeric inverse mold and replicated in arbitrary multiple material and stiffness gradients, and an array of modified geometries. Control of geometry via deformation of the inverse mold and control of stiffness via prepolymer mixing are discussed. New capabilities enabled by our approach include biomimetic actuation/sensor arrays with programmable biases, precisely tunable mechanical and geometric properties for optical or wetting applications, and flexible curved substrates. Indeed, flexibly anchored ciliary high-aspect-ratio nanostructures are now possible, and a proof-of-principle is described.
\end{abstract}

\section{INTRODUCTION}

Biology abounds with examples of functional structures, whose properties are unmatched in today's synthetic materials. Key features of biological structures include their dynamic nature, responsive behavior and often multi-functionality, which all comprise goals for the nextgeneration of smart artificial materials. Organisms and plants use sophisticated design strategies to achieve superior mechanical, optical, adhesive, self-cleaning, actuation and sensing capabilities. [1-9] A common feature of these largely unrelated designs is the use of fibers and high-aspect-ratio nano- and micro-structures.

Synthetic actuation/sensing at the sub-micron scale remains a challenging goal. Sensor arrays inspired by fish skin [10] and actuator arrays mimicking cilia still lack key features of selectivity, tunable geometry and sensitivity. In the current study we use a truly materials approach to develop a low-cost procedure for arbitrarily-designed actuated surfaces with highaspect-ratio nanostructures that are themselves responsive to a variety of stimuli and have a finely tuned geometry and stiffness. We first show how soft lithography fabrication can be extended to control both mechanical and geometric properties of these surface nanostructures. Furthermore, we demonstrate how to fabricate flexibly anchored high-aspect-ratio nanostructures where there exists a stiffness and materials gradient between the nanostructures and the underlying substrate, which represents a critical functional requirement of biological actuated nanostructures and sensors.

\section{EXPERIMENTAL DETAILS}

A square array of silicon nanoposts (height $=8 \mu \mathrm{m}$, diameter $=250 \mathrm{~nm}$, pitch $=2 \mu \mathrm{m}$ ) was fabricated using the Bosch process, as described elsewhere. [11,12] The silicon nanopost arrays were treated with an anti-sticking agent (tridecafluoro-1,1,2,2-tetrahydrooctyl)-trichlorosilane (Gelest Inc.) by exposure in a desiccator under vacuum overnight. 
Negative molds were produced from polydimethylsiloxane (PDMS), Dow-Sylgard 184, with a prepolymer-to-curing agent ratio of 10:1. The mixture was poured on the silicon nanopost substrate and degassed before being thermally cured for 3 hours at $70^{\circ} \mathrm{C}$. After cooling, the negative PDMS mold was gently peeled off the substrate. To produce replicas, the desired material was poured in liquid form into the negative mold wells. Once the material had solidified, the negative mold was simply peeled off. In this study most of the nanostructured replicas were made from a commercial UV-initiated one-part epoxy UVO- $114^{\mathrm{TM}}$ (Epoxy Technology).

For control of the modulus of the nanostructures, two liquid epoxy resins-Dow D.E.R. 331 and Dow D.E.R. 732 - were mixed in different proportions. The mixtures were based on 10\% weight increments of components, from $10 \%$ to $100 \%$ D.E.R. 331 . In all compositions, UV cross-linking initiator Cyracure UVI 6976 ${ }^{\mathrm{TM}}$ (Dow) was added to the mixture in a constant 5 weight $\%$ amount.

Bending stiffness of the cured epoxy mixtures was measured by four-point bend tests on 10x8x62 mm beams of cured epoxy mixtures, using a custom mechanical test system. A displacement rate of $500 \mu \mathrm{m} / \mathrm{s}$ and a maximum midpoint deflection of $3 \mathrm{~mm}$ was used. The stiffness values obtained were assigned to posts fabricated from corresponding mixtures.

Imaging of the nanostructures was performed on a Zeiss field emission Ultra55 SEM. Chemical analysis was performed on the SEM using Energy Dispersive Spectroscopy (EDS).

In hybrid material/stiffness replication, the nanopost material prepolymer (UVO-114) was poured on the PDMS negative mold before spinning on a Laurell WS-400-6NPP-LITE spin processor at $6000 \mathrm{rpm}$ for 3 minutes. PDMS prepolymer was poured over the uncured epoxy posts and cured for 3 hours at $70^{\circ} \mathrm{C}$. Alternatively, the epoxy film was partially UV cured 1-3 minutes and soft epoxy mixture containing 1:1 UVO-114 and D.E.R. 732 was poured on and fully UV cured for 30 minutes.

\section{RESULTS AND DISCUSSION}

We have adopted and significantly extended the soft lithography method to allow the fabrication of a biomimetic array of stable, high-aspect-ratio features. In our approach, PDMS is not the final nanostructured material; it serves as a secondary elastomeric mold for casting a derivative replica in the material of choice. Hence the stability and stiffness of the replicated structures can be controlled by appropriate material selection, and the geometry of the nanostructures can be finely tuned by applying specific deformations to the PDMS mold during replication.

The fabrication procedure is illustrated in Figure 1. The initial high-aspect-ratio master can be either formed by standard lithographical techniques, grown bottom-up (for example, nanowires) or a biological sample. We form a negative mold of the master structure in PDMS or paraffin (Figure 1B-D, N), which has an array of wells into which the desired material (polymer, liquid metal or ceramics) is cast in liquid form and cured (Fig. 1O). The mold is then either peeled off (PDMS, Figure 1P) or heated and dissolved (paraffin) to release the replicated structure. Figure 1Q shows an epoxy nanoarray that reproduces the original master with nanometer-scale resolution. Using this method one can form replicated nanostructures from polymers (e.g., epoxy, PP, PE, PMMA, PDMS, various hydrogel and shape memory polymers) and metals and alloys which have a low melting point (e.g., Ga, InBi and Woods alloy). 


\section{Controlling the geometry of nanostructures.}

One breakthrough of our approach is that the replication no longer need be one-to-one. Instead, geometric transformations from the master to the replica are easily achieved, as shown in Figure 1E-L. With liquid replica material in the PDMS negative mold, we can precisely stretch or compress the mold to transform the original 2D square lattice to a rectangular or rhombic lattice and the original circular cross-sections of the nanoposts to elliptical. By deforming the mold in the general $[h k 0]$ direction, a parallelogram unit cell with finely-tuned parameters can be formed. Tilted structures can be formed by applying a shear deformation to the mold; curved surfaces with different radii of curvature, very similar to echinoderm skin, were also fabricated (Figure 1H, 1L). In fact, any combination of the deformation types can be applied, such as the shear and $45^{\circ}$ extension in Figure 1M. This is a powerful technique to fabricate nanostructures unattainable by conventional lithographic processes.

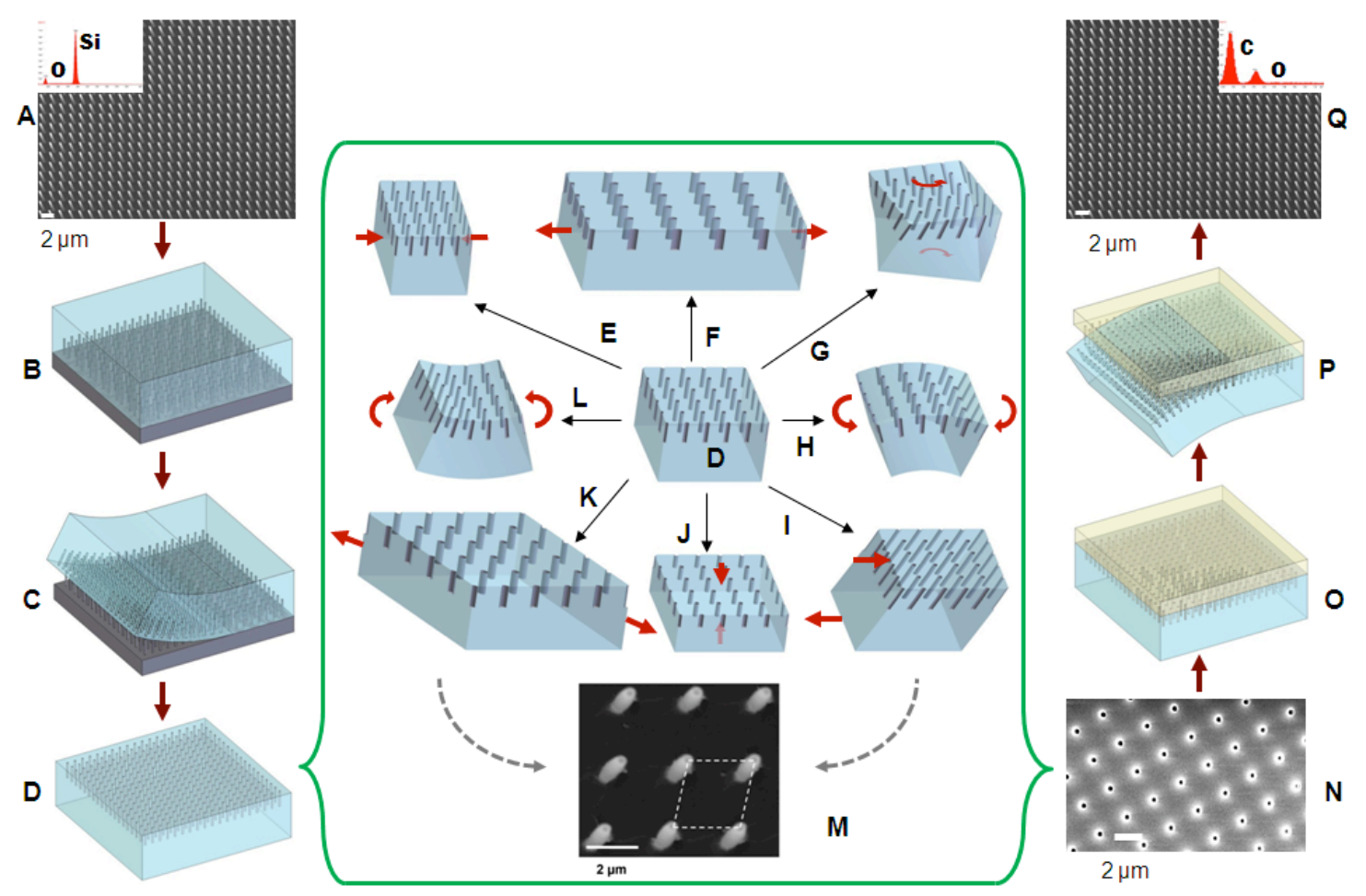

Figure 1. The fabrication procedure: casting a negative elastomeric mold from the master (A-D, N); geometric control of the replica via negative mold deformations (E-L), which can be compounded (M); pouring and casting of the replica from replica material in liquid form with arbitrary stiffness and other material properties (O-Q).

\section{Controlling the stiffness.}

To regulate micro/nanostructure sensitivity, we are able to vary their stiffness from a few MPa to hundreds of GPa by fabricating replicas in selected polymers and metals/ceramics, respectively. Furthermore, the stiffness of the array can be finely tuned by mixing in different ratios two prepolymers that exhibit high and low post-cure stiffness. To demonstrate this principle, we used two epoxy-based polymers: one with a post-cure high modulus and another with a post-cure low modulus. We were able to produce epoxy structures with a stiffness 
ranging from $\sim 1 \mathrm{MPa}$ to $\sim 1 \mathrm{GPa}$, shown in Figure 2A. The histogram can serve as a calibration curve to access any required stiffness in the $\mathrm{MPa}-\mathrm{GPa}$ range.

To increase the sensitivity of the nanoarray, we can reduce the radius (which scales as a power of four), increase the length (cubic power), and decrease the modulus (linear dependence). [13] The force needed to bend nanoposts as a function of different parameters is shown in Figure 2B. As both geometrical and material parameters have an effect on the force needed to actuate the posts, it is very helpful to introduce a unified "effective stiffness" parameter, $S_{\text {effect }}$, to directly compare the different cases. We chose to define this parameter as the force per unit deflection of the posts: $S_{\text {effect }}=F / \delta$, where $\delta$ is the deflection. In order to compare two structures, we take the ratio of the two $S_{\text {effect }}$. For a circular cross-section:

$$
\frac{S_{1 \text { effect }}}{S_{2 \text { effect }}}=\left(\frac{E_{1}}{E_{2}}\right)\left(\frac{l_{2}}{l_{1}}\right)^{3}\left(\frac{r_{1}}{r_{2}}\right)^{4}
$$
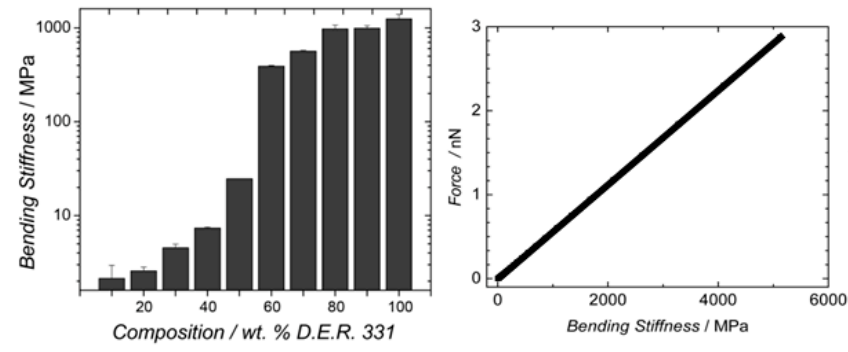

A

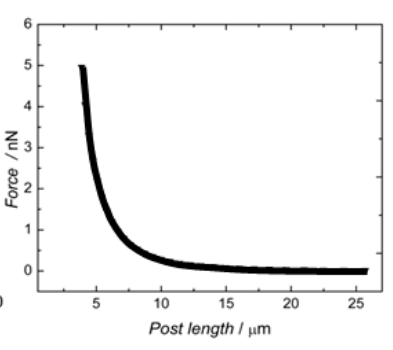

B
(1)

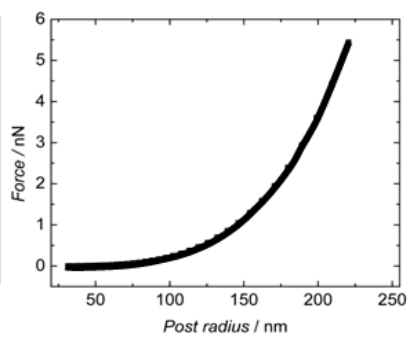

Figure 2. (A) Three orders of stiffness magnitude are accessible via simple prepolymer mixing; (B) Variation of force needed to bend cylindrical posts as a function of material stiffness, post length, and post radius.

\section{Hybrid material stiffness.}

Beyond monolithic replicas comprised of a single material and stiffness, we have further extended our method to enable multi-material replicas (Figure 3). The nanostructures of the replica may be fabricated from one replica material, while the bulk substrate is fabricated from a different material, with an optional one or more interlayers. The most interesting configuration that we are refining is rigid nanostructures attached to a soft, flexible substrate. Replica prepolymer is drop cast onto the negative mold, allowed to imbibe into the wells, and the excess is removed by spinning several minutes at high speed. At this point, a secondary intermediatestiffness layer (such as soft epoxy) can be spin coated on, or the soft bulk substrate-PDMS prepolymer in our case - can be directly cast on and cured together with the rigid nanostructures. We are working on fine gradient control between the bulk and nanostructure materials, for example by varying crosslinking of the rigid structure material before interdiffusion with the soft substrate material and by changing the temperature-time cure process for the soft substrate material.

Biological structures such as the superficial neuromasts of fish depend on stiffness gradients and anisotropy, pointing to important applications of these nanoarrays in flow sensors and as sophisticated artificial cilia. The higher elastic range of motion allowed by flexible anchoring points also suggests that such nanostructures are mechanically more robust than monolithic analogues - a hypothesis we are now studying. Flexibility of the anchoring points is demonstrated by solvent evaporation-driven clustering of stiff monolithic, hybrid-stiffness, and soft monolithic nanoposts in Figure 4. 


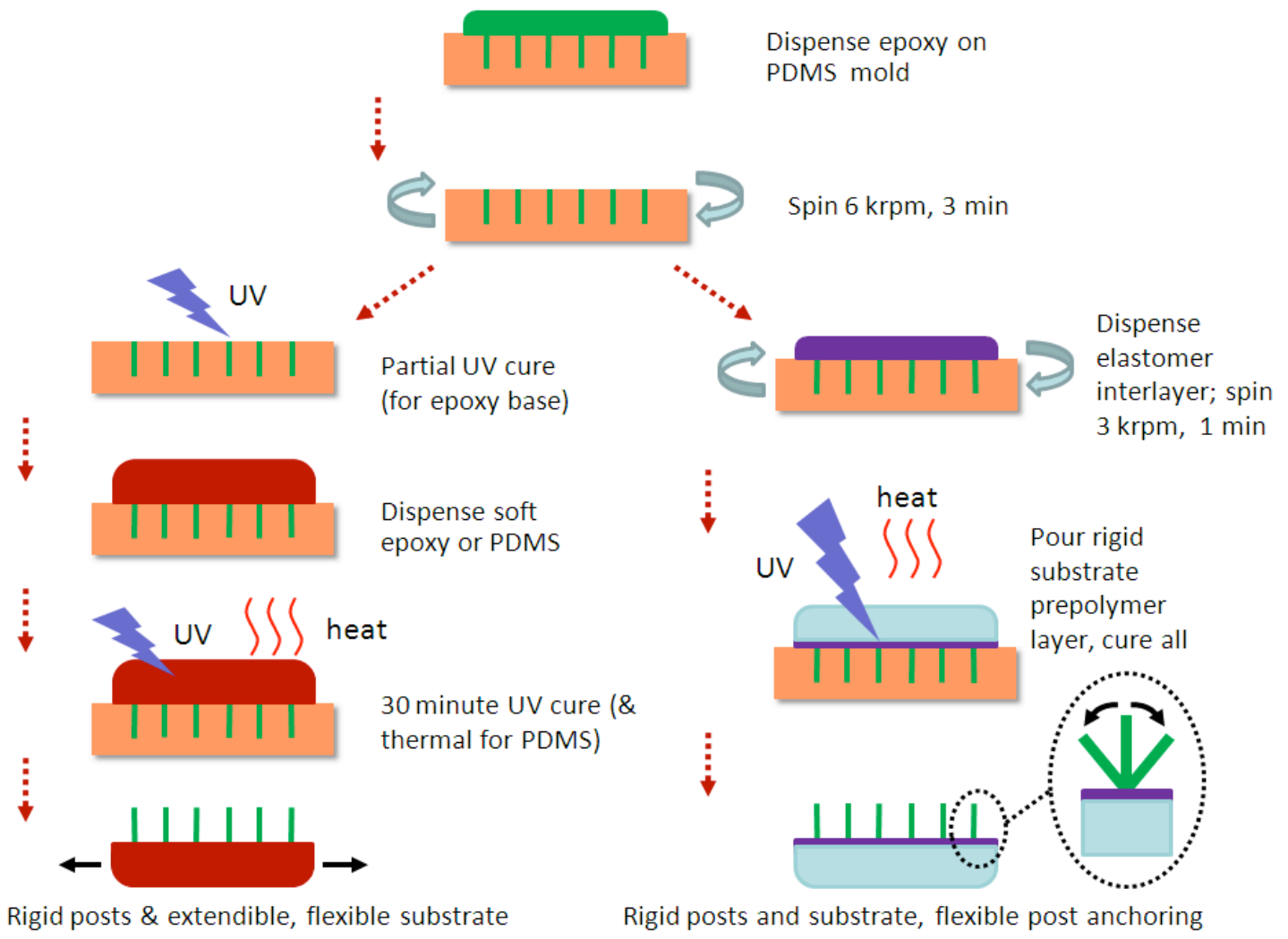

Figure 3. Fabrication of hybrid replicas that incorporate multiple materials of variable stiffness and introduce functional stiffness gradients in the system. Rigid posts may be directly attached to elastomeric substrates (Left), or they may be attached to an elastomeric thin layer, leading to flexible post anchorings on a rigid substrate (Right).

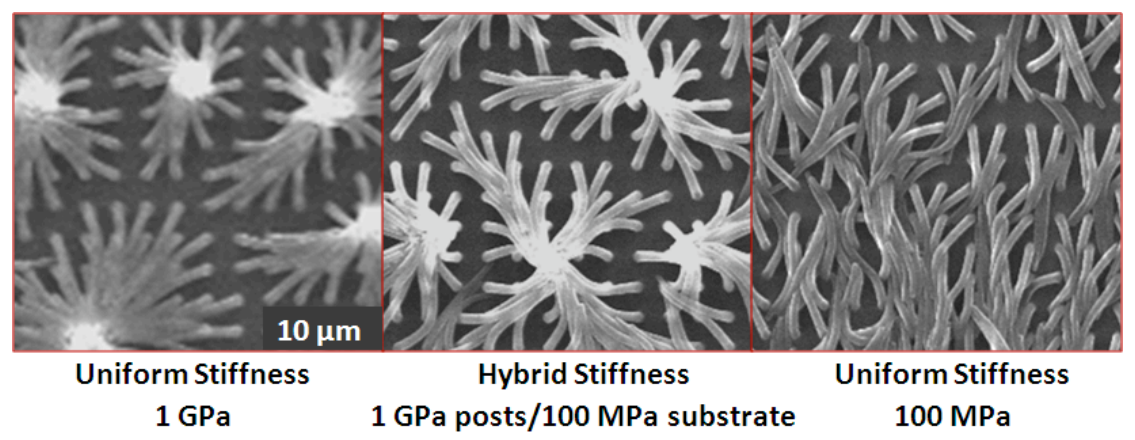

Figure 4. Anchoring point flexibility is seen in the hybrid stiffness nanoarray replica (Center), in which rigid posts are able to elastically hinge at their soft attachment points. The hybrid posts can tilt more than the monolithic rigid system (Left) but do not bend above the attachment point as in the all-soft system (Right).

Meanwhile, we have fabricated a proof of concept for mechanically actuated rigid epoxy micro/nanostructures attached to a flexible PDMS substrate. In Figure 5 we imaged the sample on an optical microscope under continuous, cyclic uniaxial extension and tracked the motion of the rigid posts relative to one another. The unit cell was extended $60 \%$ in the load direction, with a corresponding Poisson contraction. Moreover, this novel hybrid replica was extremely flexible and contour-fitting, as also seen in Figure 5. 


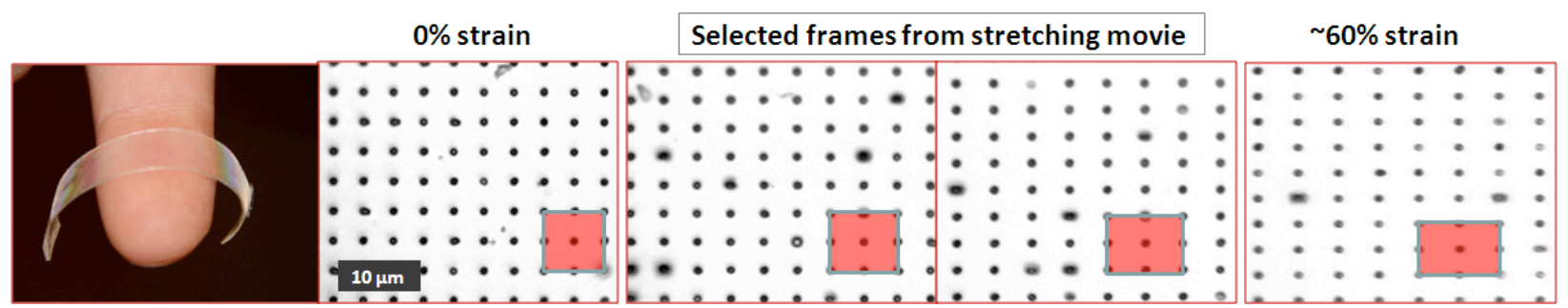

Figure 5. Mechanical in-plane actuation of micro/nanoscale rigid posts that are attached to a flexible, extendible elastomeric substrate, strained on a motorized stage. The sample is shown fitting the contour of the author's finger at left.

\section{CONCLUSIONS}

We have shown that we can produce versatile high-aspect-ratio nanostructured surfaces inspired by the echinoderm skin, gecko foot and superficial neuromasts in fish and amphibians. For this purpose, we have developed a soft-lithographic method that not only allows the one-toone replication of nanostructures with high-aspect-ratios in a variety of materials, but also makes it possible to produce nanostructures with new nontrivial cross-sections, orientations, and curved lattices - infeasible by expensive conventional lithography. This method is the only one to our knowledge that provides such a high degree and range of tunability. The resulting bio-inspired surfaces offer multifunctional characteristics that include superhydrophobicity/philicity, actuation and sensing capabilities. We believe that these structures will find exciting applications as submicron sensors, actuators and other smart materials.

\section{ACKNOWLEDGMENTS}

We would like to thank Prof. J. J. Vlassak for the use of the 4-point flexure apparatus. This work was partially supported by the Materials Research Science and Engineering Center (MRSEC) of the National Science Foundation under NSF Award Number DMR-0213805.

\section{REFERENCES}

1. W. Barthlott, C. Neinhuis, Planta, 202, 1 (1997).

2. R. Ruibal, V. Ernst, J. Morphol. 117, 271 (1965).

3. K. Autumn, S. T. Hsieh, D. M. Dudek, J. Chen, C. Chitaphan, R. J. Full, J. Exp. Biol. 209, 260 (2006).

4. J. Aizenberg, V. C. Sundar, A. D. Yablon, J. C. Weaver, G. Chen, Proc. Natl. Acad. Sci. U. S. A. 101, 3358 (2004).

5. V. C. Sundar, A. D. Yablon, J. L. Grazul, M. Ilan, J. Aizenberg, Nature, 424, 899 (2003).

6. M. J. McHenry, S. M. van Netten, J. Exp. Biol. 210, 4244 (2007).

7. J. Montgomery, S. Coombs, Brain Behav. Evol. 40, 209 (1992).

8. E. E. Ruppert, R. S. Fox, R. B. Barnes, Invertebrate Zoology, (Brooks Cole Thomson, Belmont, CA, 2004) p. 175.

9. G. Huber, H. Mantz, R. Spolenak, K. Mecke, K. Jacobs, S. N. Gorb, E. Arzt, Proc. Natl. Acad. Sci..U. S. A. 102, 16293 (2005). 
10. S. Peleshanko, M. D. Julian, M. Ornatska, M. E. McConney, M. C. LeMieux, N. Chen, C. Tucker, Y. Yang, C. Liu, J. A. C. Humphrey, V. V. Tsukruk, Adv. Mater. 19, 2903 (2007). 11. T. N. Krupenkin, J. A. Taylor, T. M. Schneider, S. Yang, Langmuir, 20, 3824 (2004).

12. S. A. McAuley, H. Ashraf, L. Atabo, A. Chambers, S. Hall, J. Hopkins, G. Nicholls, J. Phys. D: Appl. Phys. 34, 2769 (2001).

13. A. R. Ragab, S. E. A. Bayoumi, Engineering Solid Mechanics: Fundamentals and Applications (CRC Press, Boca Raton, FL, 1998), p. 944. 